\title{
Real-time Risk Assessment Framework for Unmanned Aircraft System (UAS) Traffic Management (UTM)
}

\author{
Ersin Ancel, Francisco M. Capristan†, and John V. Foster ${ }^{\ddagger}$ \\ NASA Langley Research Center, Hampton, VA, 23681, USA \\ Ryan Condotta ${ }^{\S}$ \\ Analytical Mechanics Associates, Inc., Hampton, VA, 23666, USA
}

\begin{abstract}
The new Federal Aviation Administration (FAA) Small Unmanned Aircraft rule (Part 107) marks the first national regulations for commercial operation of small unmanned aircraft systems (sUAS) under 55 pounds within the National Airspace System (NAS). Although sUAS flights may not be performed beyond visual line-of-sight or over nonparticipant structures and people, safety of sUAS operations must still be maintained and tracked at all times. Moreover, future safety-critical operation of sUAS (e.g., for package delivery) are already being conceived and tested. NASA's Unmanned Aircraft System Traffic Management (UTM) concept aims to facilitate the safe use of low-altitude airspace for sUAS operations. This paper introduces the UTM Risk Assessment Framework (URAF) which was developed to provide real-time safety evaluation and tracking capability within the UTM concept. The URAF uses Bayesian Belief Networks (BBNs) to propagate off-nominal condition probabilities based on real-time component failure indicators. This information is then used to assess the risk to people on the ground by calculating the potential impact area and the effects of the impact. The visual representation of the expected area of impact and the nominal risk level can assist operators and controllers with dynamic trajectory planning and execution. The URAF was applied to a case study to illustrate the concept.
\end{abstract}

\section{Nomenclature}

$\begin{array}{ll}A_{c} & \text { Casualty Area } \\ C_{D} & \text { Drag Coefficient } \\ E_{c} & \text { Expected Casualty } \\ \gamma & \text { Impact Angle } \\ H_{p} & \text { Height of Person } \\ P_{I} & \text { Probability of Impact } \\ L_{\text {uav }} & \text { UAV Length } \\ R_{p} & \text { Radius of Person } \\ \rho & \text { Air Density } \\ \rho_{\text {pop }} & \text { Population Density } \\ S & \text { Reference Vehicle Area } \\ W & \text { Vehicle Weight } \\ w_{\text {span }} & \text { Wing span }\end{array}$

\footnotetext{
*Aerospace Engineer, Aeronautics System Analysis Branch, 1 N Dryden St, MS 442, Hampton, VA 23681, AIAA Member.

$\dagger$ Aerospace Engineer, Aeronautics System Analysis Branch, 1 N Dryden St, MS 442, Hampton, VA 23681, AIAA Member.

$¥$ Aerospace Engineer, Flight Dynamics Branch, 8 Langley Blvd, MS 308, Hampton, VA 23681, AIAA Associate Fellow.

$\S$ Software Developer, Aeronautics System Analysis Branch, 1 N Dryden St, MS 442, Hampton, VA 23681, AIAA Member.
} 


\section{Introduction}

TnMANNED aircraft systems (UAS) have been the focus of many commercial and civilian applications including infrastructure monitoring, delivery of goods, precision agriculture, public safety, search and rescue, disaster relief, weather monitoring, among many others. However, the implementation of such commercial operations has been slow. The large-scale small UAS (sUAS) applications are hampered by lack of airspace operations requirements, procedures, and support functions ${ }^{1}$ as well as privacy considerations, public acceptance, and environmental concerns. As the demand for sUAS is projected to increase dramatically, the Federal Aviation Administration (FAA) published Small Unmanned Aircraft Regulations (Part 107) ${ }^{2}$ that describe the operating requirements for commercial drone use. The Part 107 regulations state that unmanned aircraft weighing less than $55 \mathrm{lb}$ can operate within visual line of sight (VLOS) and below 400 feet above ground; however, operations are prohibited over populated areas. Contrary to these regulations, many commercial use cases necessitate beyond visual line of sight (BVLOS) operations over the general population to achieve full benefits (e.g., infrastructure inspection or parcel delivery). In order to enable the path to VLOS and BVLOS autonomous vehicle operations, NASA initiated the UAS Traffic Management (UTM) project. The UTM concept is a systematic approach to safely accommodate all manned and future UAS operations within low-altitude airspace by providing services like airspace design, corridors, dynamic geo-fencing, severe weather and wind avoidance, congestion management, terrain avoidance, route planning, re-routing, separation management, sequencing, spacing, and contingency management. ${ }^{3}$ NASA collaborates with industry, the FAA, and several other government agencies to define roles and responsibilities of primary entities within the UTM ecosystem. The acquired capabilities are tested and showcased via Technology Capability Level (TCL) flight demonstrations.

Within the UTM project, the UTM Safety \& Risk Group based at NASA Langley Research Center (LaRC) is focused on the determination of potential current and future safety hazards, the development of UTM safety case and test scenarios, the formulation of off-nominal trajectory and impact point prediction methods, as well as the development of a real-time risk assessment approach in order to satisfy UTM's safe airspace operations and public safety requirements. This paper addresses the need for a real-time risk assessment approach in UTM by providing a real-time risk assessment framework that quantifies the risks to bystanders due to sUAS especially for operations in populated areas.

This research was built upon the well-studied subject of third-party risk associated with UAS operations. Third party risk research considers the risk to the people on the ground, who are not directly involved with the aircraft operation. Within the same context, Lazatin ${ }^{4}$ and Lum \& Waggoner ${ }^{5}$ estimated insurance liability for various UAS use cases based on fatalities due to ground and mid-air collisions by employing population density, population distribution, and sheltering factors. Similarly, Aalmoes et al. ${ }^{6}$ developed a conceptual third party risk model that estimates the potential impact area and the consequence area. Using a different approach, Clothier et al. ${ }^{7}$ assigned various UAS type categories based on kinetic energy and impact severity (injury or fatalities). Lum et al. ${ }^{8}$ and Ford \& McEntee ${ }^{9}$ simulated the impact area based on initial failure location and used satellite imagery and census information to estimate the number of collisions with a bystander per flight hour. Additionally, Dalamagkidis et al., ${ }^{10}$ Weibel \& Hansman, ${ }^{11}$ and Burke et al. ${ }^{12}$ employed similar methods to estimate casualties and approximate the effects of kinetic energy to drive the target ground impact frequency based on population density. Finally, Melynk et al. ${ }^{13}$ compared past studies, utilized similar methods to enable casualty prediction, and also attempted to validate their model using fatality rates caused by general aviation crashes, obtained from historical data. The literature review also revealed that a dynamic methodology, capable of providing real-time risk to the population on the ground, had not yet been attempted.

In order to assist UTM safety goals, this research proposes a modular methodology, named UTM Risk Assessment Framework (URAF), that can provide risk metrics associated with casualties in real-time. The novel approach adopted in this research includes the use of dynamic aircraft health and environmental data to provide real-time mishap likelihood by using Bayesian Belief Networks (BBNs). Section II provides an overview of the URAF components while Section III presents a simulated case study to demonstrate the framework capabilities. Future work and conclusions are given in Sections IV and V, respectively. 


\section{UTM Risk Assessment Framework (URAF)}

Given their flexibility and envisioned use cases, small unmanned aircraft are expected to operate within close proximity to people and property on the ground. The proposed risk assessment framework provides the foundation for a tool that can be used to estimate risk associated with sUAS flights. The resulting tool is envisioned to assist the operators or traffic controllers by displaying real-time risk based on actual aircraft status and operating environment. The framework is comprised of various sections that estimate and display the operational risk, which is comprised of the likelihood of undesirable events and their impacts, as highlighted in Fig. 1.

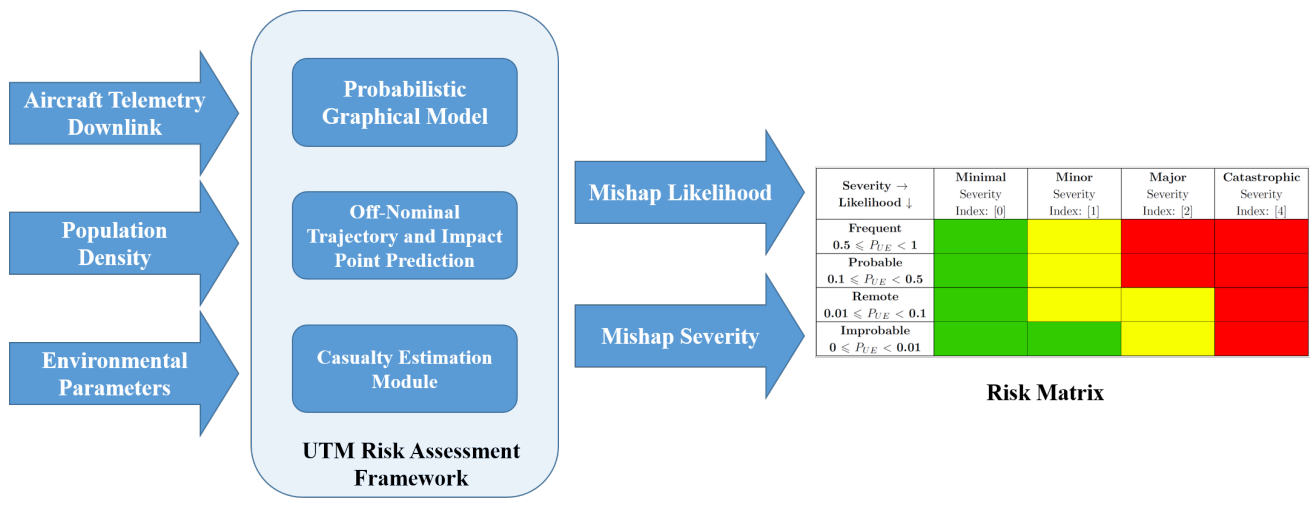

Figure 1. URAF Architecture Components.

In order to obtain real-time flight risk, the framework requires dynamic data from internal and external parameters, where available. These parameters include aircraft system status data provided by on-board health monitoring, environmental variables such as wind or other factors obtained by aircraft sensors and/or ground services, and population density and distribution, which are essential to estimate casualty values given the aircraft's ground collision. Several commercial off-the-shelf (COTS) flight controller hardware in the market are capable of accessing and transmitting vital aircraft health status along with wind information to the ground control station. Additionally, it may be feasible to obtain dynamic population density and distribution by considering cellular network tower data as proxy. Once the data stated above are gathered on the ground control station, they are utilized by three separate modules; where

- The Probabilistic Graphical Model estimates the likelihood of predetermined mishaps using data from aircraft health systems status as part of the aircraft telemetry downlink,

- The Off-Nominal Trajectory and Impact Point Prediction module provides an expected impact point for the modeled mishap with an associated uncertainty bound, and

- The Casualty Estimation module employs the impact point and associated uncertainty as well as population distribution data to evaluate injury or fatality to humans on the ground.

These modules work in concert to continuously evaluate the likelihood and the consequences of the designated mishaps, effectively providing the real-time risk severity values, which are periodically updated and displayed on the ground control station computer. It is important to note that, thanks to its modular structure, the URAF is envisioned to be the backbone for an upcoming suite of tools, built upon each other with increasing fidelity and capabilities. The next sections provide further details on these modules.

\section{A. Probabilistic Graphical Model}

In order to examine the safety implications of operating sUAS within the UTM operational environment, adequate representations of mishaps and their causal and contributing factors need to be developed. As previously noted, the Probabilistic Graphical Model provides the likelihood of mishap occurrence by fusing real-time aircraft system data with detailed mishap models. To develop these models, an analysis of current and future sUAS operations was performed by reviewing UAS mishap reports and future use cases, as highlighted by Belcastro et al. ${ }^{3}$ Identified hazards as well as their causal and contributing factors were then 
used to construct the event sequence diagrams (ESDs), which are converted to probabilistic models. The Bayesian approach was chosen to graphically represent and model the mishaps considered within the URAF framework.

\section{Bayesian Approach}

The URAF employs the flexible, probabilistic approach of Bayesian belief networks (BBNs) to model and estimate the probability of the predetermined UAS mishaps. The BBN is a directed acyclic graphical representation of a framework where causal factors and outcomes are linked to each other to form a network. Each element of the graphical structure is associated with an initial probability distribution, acquired either empirically or subjectively. ${ }^{14,15}$

One of the major advantages of using a Bayesian approach to interpret probability is that it allows modelers to revise and adjust predictions of the causal factors and observe the outcomes in the light of new data. ${ }^{16}$ In other words, the initial subjective assumptions can be replaced by perceived evidence and the network will output the updated mishap probability given the presence of such evidence. Within the URAF, this capability allows the insertion of the real-time aircraft health monitoring data to the model and the revision of the likelihood of mishap occurrence.

Another advantage of using the Bayesian approach is that the models are capable of taking into consideration both objective and subjective measures of uncertainty. ${ }^{17,18}$ Compared to their manned counterparts, input data for UAS operation safety assessments contain significant sources of uncertainty. These uncertainties include: a) the lack of UAS operational data and experience, b) use of non-regulated COTS components, and c) a vast range of UAS configurations (multi-rotor, fixed wing, and hybrid setups), which present challenges to identification, collection, and assessment of aircraft failure modes and their impacts. Consequently, Bayesian methods' ability to operate with subjective data due to insufficient operational experience render the approach well-suited for quantitative UAS modeling. The Bayesian approach has also been extensively used to represent and evaluate uncertainty in aviation safety applications in addition to a large number of other complex reliable systems, such as the nuclear power industry, cyber security, space launch industry, etc. ${ }^{14-19}$ Assessing the operational risk of flying unmanned aircraft over populated areas requires a systems perspective approach that considers the system to be a series of interconnected and interacting parts, together delivering some desirable outcome and unintended consequences. ${ }^{16}$

\section{Model Development}

As discussed above, within URAF, the probabilistic representation of undesirable events (e.g., CFIT, MAC, uncontrolled crash, etc.) is performed by BBNs. The model development process was initiated with the identification of undesirable events, respective causal factors, and their dependencies. Next, aircraft system failures, their propagation patterns, and combined effects of multiple failures were constructed using event trees, fault trees and/or failure modes \& effects analysis (FMEA), all of which can be replicated using the BBNs. ${ }^{16}$ For this research, the Hugin Developer v.8.4 ${ }^{20}$ was selected as the BBN modeling software since it allows application program interface (API) for $\mathrm{C}, \mathrm{C}++$, Java, and .Net. The program interface capability enables data flow between the aircraft telemetry stream and the other complementing modules (i.e., Impact Point Predictor given in Section II.B, and Casualty Estimation Module provided in Section II.C). Additionally, Hugin software capabilities and suitability for aviation safety research were previously highlighted in Ancel et al., ${ }^{14}$ Luxhoj, ${ }^{15}$ and Ancel and Shih. ${ }^{21}$

The hierarchy within the Bayesian networks is expressed in a manner similar to a family tree where child node probabilities are influenced directly by those of its parent nodes. The probability of each child node and the effects of the parent nodes are represented via a conditional probability table (CPT). ${ }^{14,15}$ Barr et al. ${ }^{22}$ which focused on developing a preliminary risk assessment approach, highlights a generic UAS mishap $\mathrm{BBN}$ development and data population processes in detail.

\section{Data Population}

Following the development of the undesirable event model, the next step involves the collection and population of the data. As highlighted by Aalmoes et al., ${ }^{6}$ given that unmanned systems are in their early development stages, operational data needed to populate third party casualty models are lacking. Consequently, the data necessary to drive numerous risk models were approximated through various sources such 
as numerical distributions, ${ }^{6,23}$ manned aircraft failure rates, ${ }^{4,6,11}$ and/or military unmanned or manned operational data. ${ }^{10}$ The authors believe that, although such assumptions might be suitable for approximations or comparative studies of various future operational concepts, reliability data from manned aircraft operations do not provide an adequate baseline due to vast redundancy and configuration differences between manned and unmanned aircraft as well as the varying operational context (military vs. civilian aircraft). Additionally, limited operational and empirical sUAS failure data mandates the utilization of expert provided subjective values for conditional probability assessment. By utilizing dynamic aircraft health data, the methodology suggested in this paper provides an alternative, and perhaps more suitable, approach for risk assessment while overcoming the limitations due to lack of empirical data. Consequently, taking advantage of the real-time aircraft health monitoring data not only enables a dynamic risk assessment capability but also helps alleviate the reliance on subjective data for component/system reliability rates.

\section{B. Off-Nominal Trajectory and Impact Point Prediction Module}

An important part of the URAF framework is the prediction of flight trajectories and/or ground impact point due to a severe off-nominal event. For the purposes of this discussion, an off-nominal event is defined as a significant deviation from the intended flight trajectory or a loss of control resulting in extreme vehicle attitudes (pitch, roll) exceeding the normal flight envelope. Thus, as a result of an off-nominal event, the risk of undesirable events such as impact with people or a stationary object (building) increases and is therefore a required consideration for risk assessment.

Prediction of off-nominal trajectories presents significant challenges due to the many possible failure scenarios and the wide range of vehicle configurations that operate under the UTM framework. One of the objectives of the UTM project is the development of modeling and simulation methods that would allow prediction of flight dynamics behaviors and trajectories suitable for risk analysis as well as implementation in a real-time risk assessment tool. ${ }^{24-26}$ Due to practical considerations, low-order, generic modeling methods were sought since they offer the potential for rapid solutions and easier implementation.

Currently little has been published regarding high-fidelity modeling of sUAS vehicles in off-nominal conditions. Modeling and simulation of multi-rotor vehicles is now the subject of research, but validated experimental data is limited. To date, trajectory prediction has been limited to point-mass or ballistics methods, which are generally applicable only to complete power failure or degraded control configurations. Lum et al. ${ }^{8}$ simulated a large number of failures and observed the dynamics of the powerless aircraft to determine the impact zones and areas from these failures. The research concluded that vast majority of crashes occur close to the initial failure location. Similarly, Aalmoes et al. ${ }^{6}$ attempted to represent the potential impact area as a function of the aircraft weight, speed, direction and maximum glide distance. Recent research by Foster \& Hartman ${ }^{24}$ provided simulation predictions of multirotor trajectories during propulsion failures. The research results showed the prediction of a tumbling mode during abrupt motor failure and the resulting near-ballistic trajectory. In addition, a cascading power failure was shown to cause erratic transition dynamics as the vehicle entered into an out-of-control descent.

One example of real-time trajectory prediction for sUAS vehicles is the flight termination system used for the NASA AirSTAR flight test facility. ${ }^{27}$ In the event of a flight anomaly requiring termination, the system was designed to put the vehicle in a sustained high angle of attack/high drag condition using prospin controls in order to create a near-vertical but predictable trajectory. An Impact Point Prediction (IPP) algorithm was developed to estimate this trajectory from initiation of termination to ground impact, based on the predicted rate of descent and known winds. An additional feature was an estimation of the transition dynamics from forward flight to the sustained descent. The algorithm was designed to provide impact distance accuracy of $10 \%$ of the initial altitude at termination. The IPP algorithm was implemented in a real-time Monte Carlo fashion in which important parameters such as total drag coefficient, wind direction and speed, and transition parameters were used as dispersion parameters. The 2-sigma potential impact area was simply computed from the intersection of the trajectory profiles with the known ground elevation. A limitation of this method is the assumption that the vehicle can achieve and maintain the high-drag condition as well as assumptions regarding the transition dynamics. Regardless of the method of choice, a high accuracy prediction of the impact point plays a crucial role in predicting risks to bystanders via the Casualty Estimation Module (discussed in the next section). 


\section{Casualty Estimation Module}

Following the estimation of the impact point and potential impact area, the next step in evaluating the sUAS risk is to determine the effects of the aircraft crash on the population below. Although financial impacts of UAS crashes can be substantial, ${ }^{5}$ (e.g., UAS replacement value, repair of damage to property, environmental cleanup costs, etc.) within the URAF methodology only the human safety or loss of life was considered as the risk assessment metric.

As previously discussed, a large number of studies have estimated risks associated with UAS operations over populated areas. These studies considered several parameters to estimate casualties. These parameters include population density and distribution, sheltering effects, casualty impact area (also called debris field or risk exposure area) and impacts of kinetic energy on casualty severity given the aircraft impact. Melynk et al. ${ }^{13}$ provided a through comparison of various approaches and examined the nuances and assumptions for these parameters. The comparison revealed that one of the most critical but varying parameters was related to the estimation of casualty area. The casualty area (or the hypothetical area where casualties are expected following an aircraft impact) along with the population density parameter is used to determine the number of people exposed to risk on the ground.

Based on a literature review of well-known metrics, expected number of casualties, often referred as $E_{c}$, was selected as the metric used to quantify the risk to bystanders in the URAF methodology. From a decision making perspective, the expected number of casualties is an appealing metric because it provides a single measure of safety and can be adjusted to provide casualty estimates given a specific scenario (conditional expectation). This metric has also been used widely in the range safety analysis of launch and reentry vehicles, ${ }^{28,29}$ and thus it is well understood. A simple expression of the expected number of casualties given that an object impacts a certain location takes the following form:

$$
E_{c}=\rho_{\text {pop }} A_{c}
$$

where $\rho_{\text {pop }}$ represents the population density and $A_{c}$ represents the casualty area. This expression highlights the importance of estimating population density and the casualty area for even the simplest version of the expected number of casualties equation. Population density estimates are often derived from census data, while the casualty area is often a function of the vehicle shape and the impact trajectory. The use of the $E_{c}$ formulation along with the consideration of casualty area and sheltering effects are discussed in more detail in Section III.D.

\section{Risk Construct}

In order to determine and visualize the flight risk, the levels and boundaries of probability of mishap occurrence as well as severities of the mishap need to be established. The modeled mishap likelihood and associated severities are combined to reflect the resultant risk, which is represented using a risk matrix, originally introduced in Military Standard (MIL-STD) 882. ${ }^{30}$ The rows in the matrix reflect mishap likelihood and the columns provide severity categories where intersection of the two signifies the associated risk of the failure or mishap. The URAF methodology uses a modified version of the risk matrix developed for unmanned aircraft systems, which was cited within FAA's Safety Management System Manual, ${ }^{31}$ to establish and visualize real-time UAS flight risks.

\section{Undesirable Event Likelihood}

Likelihood is defined as the estimated probability or frequency, in quantitative or qualitative terms, of a hazards effect or outcome. ${ }^{31}$ In order to express likelihood quantitatively, the assessment of failure rates is performed individually for each failure condition or mishap upon the basis of relevant experience, which can be acquired through a combination of data from testing, modeling and simulation, expert judgement, and structured analysis techniques. ${ }^{18,30}$ Barr et al. ${ }^{22}$ suggests employing allowable small aircraft hazard probabilities mandated by FAR Part 23 requirements; however, the range of allowable hazard probabilities is dependent upon the aircraft and the allowable risk levels and is still the subject of research.

Given that the URAF methodology outputs mishap likelihood values derived from aircraft telemetry data, the authors propose employing these probability values instead of approximating failure rates per flight hour. Based on simulated case studies and data acquired from UAS hazard identification efforts, a modified version of the likelihood scale given by Haimes ${ }^{30}$ was adopted for the current iteration of the framework (Table 1). It 
is important to note that the accepted likelihood values require continuous revision and refinement as more operational data associated with sUAS flight risks become available in the future.

Table 1. Undesirable Event Probability Levels.

\begin{tabular}{c|c|c|c} 
Improbable & Remote & Probable & Frequent \\
\hline $0 \leqslant P_{U E}<0.01$ & $0.01 \leqslant P_{U E}<0.1$ & $0.1 \leqslant P_{U E}<0.5$ & $0.5 \leqslant P_{U E}<1$
\end{tabular}

\section{Undesirable Event Consequences}

Similar to the likelihood discussion above, the consequences of undesirable events need to be determined and ranked in order to construct the risk reference system for URAF. The FAA Safety Management System (SMS) manual defines severity as the consequence or impact of a hazard's effect or outcome in terms of degree or harm. Consequently, the severity scales, often described qualitatively and quantitatively, range from minimal discomfort to those on the ground to fatality or fatal injury to bystandersbased on type of hazards. ${ }^{18,31}$ The mishap severity classification and definitions given in FAA's SMS manual were adopted and modified to be used with URAF methodology.

The estimated consequence metrics involve a combination of $E_{c}$ and kinetic energy (KE). This combination takes place by discretizing $\mathrm{KE}$ and $E_{c}$. Weights are then associated with each discretized level and the product of the KE weight and $E_{c}$ weight provides the final consequence level. The weights associated with $\mathrm{KE}$ and $E_{c}$ are:

- Low $\mathrm{KE}=1$

- High $\mathrm{KE}=2$

- $0 \leqslant E_{c}<0.1=0$;

$\bullet 0.1 \leqslant E_{c}<0.5=1$; and

- $0.5 \leqslant E_{c}=2$

Using the product of the weights (or severity indices), the undesirable event consequences were classified as given in Table 2. For instance, Major category can be caused by both high KE coupled with $0.1 \leqslant E_{c}<$ 0.5 , and low $\mathrm{KE}$ and $0.5 \leqslant E_{c}<1$ combinations. It is important to note that the descriptions provided in Table 2 aim to tackle the main concerns in the given severity index. The probability of fatality as a function of kinetic energy (KE) has been extensively covered in the literature ${ }^{4,7,10,13}$ and was not within the scope of the URAF framework. For that reason, the consequence scale delineates between high and low KE based on the threshold determined by the method of choice. Similarly, the weight ranges can be adjusted according to mishap characteristics.

Table 2. Undesirable Event Consequences.

\begin{tabular}{c|c|c|c} 
Minimal & Minor & Major & Catastrophic \\
\hline $\begin{array}{c}\text { Discomfort to those } \\
\text { on the ground }\end{array}$ & $\begin{array}{c}\text { Non-serious injury to } \\
\text { people on the ground }\end{array}$ & $\begin{array}{c}\text { Serious injury to } \\
\text { people on the ground }\end{array}$ & $\begin{array}{c}\text { Fatality or fatal injury } \\
\text { to people on the ground }\end{array}$ \\
\hline Severity Index: $[0]$ & Severity Index: [1] & Severity Index: $[2]$ & Severity Index: [4]
\end{tabular}

\section{URAF Risk Assessment Matrix}

As previously discussed, a risk matrix is used to classify and assess the risk with known likelihood and severity. The minimum acceptable level of risk is determined by severity categories; the FAA Risk matrix ${ }^{31}$ contains three risk categories (low, medium, and high), however two-level ${ }^{5}$ or five-level $^{23}$ (high, serious, medium, low, and eliminated) scales can also be employed based on the context. Based on the likelihood and severity scales discussed above, the risk matrix given in Table 3 was used in URAF. Based on the matrix mapping, mishaps are assigned High (highlighted red, unacceptable risk), Medium (highlighted yellow, minimum acceptable risk), and Low (highlighted green, acceptable without restrictions). ${ }^{31}$ It is important 
to note that an improbable failure with catastrophic consequences was considered High risk due to numerous single point failure sources present with sUAS operations.

Table 3. URAF Risk Matrix.

\begin{tabular}{|c|c|c|c|c|}
\hline $\begin{array}{c}\text { Severity } \rightarrow \\
\text { Likelihood } \downarrow\end{array}$ & $\begin{array}{c}\text { Minimal } \\
\text { Severity } \\
\text { Index: }[0]\end{array}$ & $\begin{array}{c}\text { Minor } \\
\text { Severity } \\
\text { Index: }[1]\end{array}$ & $\begin{array}{c}\text { Major } \\
\text { Severity } \\
\text { Index: }[2]\end{array}$ & $\begin{array}{c}\text { Catastrophic } \\
\text { Severity } \\
\text { Index: }[4]\end{array}$ \\
\hline $\begin{array}{c}\text { Frequent } \\
\mathbf{0 . 5} \leqslant P_{U E}<\mathbf{1}\end{array}$ & & & & \\
\hline $\begin{array}{c}\text { Probable } \\
\mathbf{0 . 1} \leqslant P_{U E}<\mathbf{0 . 5}\end{array}$ & & & & \\
\hline Remote & & & & \\
$\mathbf{0 . 0 1} \leqslant P_{U E}<\mathbf{0 . 1}$ & & & & \\
\hline $\begin{array}{l}\text { Improbable } \\
\mathbf{0} \leqslant P_{U E}<\mathbf{0 . 0 1}\end{array}$ & & & & \\
\hline
\end{tabular}

\section{Case Study}

As part of UTM TCL demonstration efforts, a series of flights are being planned to showcase technologies developed by NASA LaRC UTM Safety team. The flights are scheduled to be performed during the Summer of 2017. In order to demonstrate the framework proposed in Section II, representative TCL flight log data was simulated for use as a case study. The following sections present the details and URAF methodology outputs for this flight.

\section{A. Flight Mission and Assumptions}

The simulation used a DJI S1000 ${ }^{32}$ octocopter that was programmed to follow an autonomous trajectory over NASA LaRC. The case study was designed to accomplish simulated safety objectives including corona detection at a LaRC power substation, a pipeline inspection, an encounter with a non-cooperative aircraft, and a low altitude flight in an urban setting. A tool that was developed based on the URAF methodology was enabled on the ground control station computer to provide real-time flight risk due to a crash following an unpowered descent, which was considered as the undesirable event. The ground control station ran the Mission Planner open source software that holds the Python script for exchanging data between programs. The Mission Planner software received MAVLINK messages via the telemetry link from the aircraft equipped with PixHawk autopilot. The aircraft state information was then processed with MATLAB and Python scripts to execute both the Hugin BBN model and off-nominal trajectory and impact point estimation subroutines. Once the impact point was calculated, the aircraft state data and the impact point data were sent to APM Planner v2.0 ${ }^{33}$ software for visualization to the user. To demonstrate varying levels of casualty risks, the simulation included system failures while the aircraft was flying over people located out in the open. The specific scenario variables are discussed in Section III.E.

The goal of the first iteration software given in this case study was to demonstrate the feasibility of the proposed URAF methodology. Consequently, the authors primarily focused on linking the aircraft provided data with the software suite and obtaining cohesion among the aforementioned modules. For that reason, several assumptions were made to ensure the minimal capability was achievable within an easily observable context. A summary of these assumptions along with planned capability improvements are given in Table 4 . 
Table 4. Case Study Assumptions and Planned Improvements.

\begin{tabular}{|c|c|c|}
\hline $\begin{array}{l}\text { URAF } \\
\text { Components }\end{array}$ & Case Study Scope \& Assumptions & Planned Improvements \\
\hline $\begin{array}{l}\text { Aircraft } \\
\text { Telemetry } \\
\text { Downlink }\end{array}$ & $\begin{array}{l}\text {-Aircraft Health Monitoring parameters } \\
\text { limited to GPS, Telemetry Health, and } \\
\text { Power System Status }\end{array}$ & $\begin{array}{l}\text {-Implementation of array of sensors } \\
\text { providing comprehensive aircraft } \\
\text { health data (servo and thrust response) }\end{array}$ \\
\hline $\begin{array}{l}\text { Population } \\
\text { Density }\end{array}$ & $\begin{array}{l}\text {-Static population density derived from } \\
\text { building characteristics and occupancy } \\
\text {-Capability to customize population } \\
\text { distribution }\end{array}$ & $\begin{array}{l}\text {-Near real-time population distribution } \\
\text { and density via census data augmented } \\
\text { by cellular network activity }\end{array}$ \\
\hline $\begin{array}{l}\text { Environmental } \\
\text { Parameters }\end{array}$ & $\begin{array}{l}\text {-Preloaded wind profiles obtained } \\
\text { from aviation weather sources }\end{array}$ & $\begin{array}{l}\text {-Reliable wind profile data obtained } \\
\text { by on-board sensors or UTM data } \\
\text { service providers }\end{array}$ \\
\hline $\begin{array}{l}\text { Probabilistic } \\
\text { Graphical } \\
\text { Model }\end{array}$ & $\begin{array}{l}\text {-Low-fidelity aircraft systems model } \\
\text { populated with arbitrary data } \\
\text {-No human factors models (operator, } \\
\text { maintenance adverse conditions) }\end{array}$ & $\begin{array}{l}\text {-Enhanced aircraft system and failure } \\
\text { mode representation populated with } \\
\text { real-time, operational and SME data } \\
\text {-Organizational and human factors } \\
\text { modeling to estimate operator and } \\
\text { maintenance related issues }\end{array}$ \\
\hline $\begin{array}{l}\text { Trajectory \& } \\
\text { Impact Point } \\
\text { Prediction }\end{array}$ & $\begin{array}{l}\text {-Single mishap profile (unpowered } \\
\text { descent/terminated flight) } \\
\text {-Descent rate equivalent to terminal } \\
\text { velocity } \\
\text {-2-sigma impact point uncertainty } \\
\text { obtained by Monte Carlo analysis }\end{array}$ & $\begin{array}{l}\text {-Multitude of off-nominal conditions } \\
\text { (mid-air collision, uncontrolled descent, } \\
\text { partial aircraft control, propulsion and } \\
\text { power failure) } \\
\text {-Dedicated trajectory model for each } \\
\text { considered mishap types }\end{array}$ \\
\hline $\begin{array}{l}\text { Casualty } \\
\text { Estimation } \\
\text { Module }\end{array}$ & $\begin{array}{l}\text {-Casualty area calculation assumes } \\
\text { vehicle glide } \\
\text {-Uniform population distribution within } \\
\text { each grid cell } \\
\text {-No secondary impact effects (e.g., fire } \\
\text { casualties due to building damage, etc.) }\end{array}$ & $\begin{array}{l}\text {-Inclusion of various secondary impact } \\
\text { effects coupled with augmented } \\
\text { population density and distribution } \\
\text { information }\end{array}$ \\
\hline
\end{tabular}

\section{B. Bayesian Belief Network Model}

As previously discussed, BBN modeling using Hugin Developer software was implemented as the probabilistic modeling method to estimate mishap likelihoods based on real-time aircraft component status. In order to do so, a simplistic model given in Fig. 2 was developed. The BBN model continuously updates the input values for GPS Count, GPS Status, Remaining Battery, Battery Voltage, Telemetry Health, and Wind Speed dependent upon the aircraft altitude. These values are then used to calculate the probability of associated system failures. The model outputs are used as both a decision making aid and as a precursor for an imminent mishap to inform the ground control station operator. The Return to Base (RTB) node provides the threshold value for executing RTB action based on minimum acceptable Navigation capability and Lost Link status. The CPT for the RTB node is populated to behave like a switch to inform the operator that the RTB execution is preferable due to evidence that the navigation system and/or command and control link may be operating below acceptable limits. The Unpowered Descent represents the modeled undesirable event based on the combined likelihood of power system failure and degraded controllability. The probability output from the BBN model is used in the likelihood axis of the risk matrix discussed in Section III.D. The aircraft mishap modeling and data population processes were previously discussed in detail by Ancel et al. ${ }^{21}$ and Barr et al. ${ }^{22}$ 


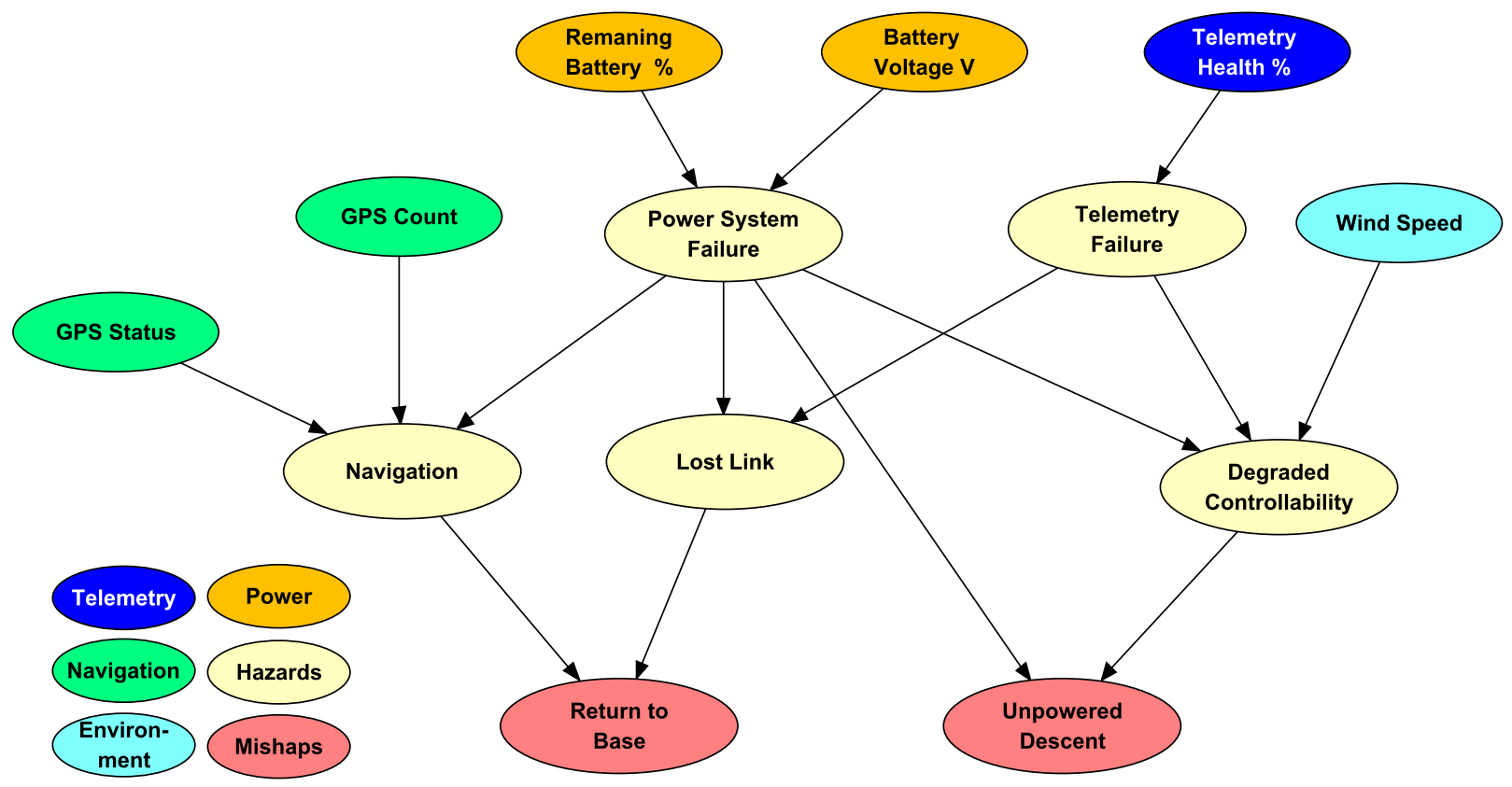

Figure 2. BBN Model.

\section{Trajectory and Impact Point Prediction}

This case study used the AirSTAR Impact Point Prediction algorithm ${ }^{27}$ previously discussed in Section II.C. Given that the purpose of this effort was to demonstrate the conceptual implementation of a real-time trajectory prediction algorithm, a high-fidelity representation of the actual off-nominal trajectory was not considered. The development of more generic and robust methods for numerous types of failures is being contemplated for future implementations of the URAF methodology.

The inputs to the algorithm included the estimated average drag coefficient for a multi-rotor vehicle in tumbling motion $\left(\mathrm{C}_{D}\right)$, air density $(\rho)$, the reference vehicle area $(S)$, and the vehicle weight $(W)$. The altitude rate of change $(\dot{h})$ was estimated with Eq.(2) where the rate of descent was assumed to be the the vehicle airspeed in a $1 \mathrm{~g}$, near-vertical flight path, which corresponds to terminal velocity. As Dalamagkidis et al. ${ }^{10}$ suggested, the use of terminal velocity (or maximum operating velocity increased by $40 \%$ as an alternative value) is probably an over-conservative estimation for small UAS flying at low altitude. However, for this case study the assumption was used for estimating kinetic energy on impact.

$$
\dot{h}=-\sqrt{\frac{W / S}{\frac{1}{2} \rho C_{D}}}
$$

The transition dynamics were modeled in an ad hoc fashion that assumed a level transition to a constant rate of descent and a first-order decay in forward ground speed. Table 5 describes the dispersion parameters and associated dispersion values to obtain a 2-sigma potential impact area. The values shown for transition time, airspeed decay time constant, and drag coefficient were based on preliminary estimates from Foster \& Hartman ${ }^{24}$ and Cunningham et al. ${ }^{27}$ 
Table 5. Dispersion Parameters for IPP Monte Carlo Algorithm.

\begin{tabular}{c|c|c} 
Dispersion Parameter & Nominal Value & Dispersion \\
\hline Transition time (sec) & 2.5 & $+/-0.5$ \\
\hline Inertial airspeed decay time constant (sec) & 2.5 & $+/-1$ \\
\hline Wind direction (deg) & Measured & $+/-10$ \\
\hline Wind speed (knots) & Measured & $+/-5$ \\
\hline Drag coefficient & 0.4 & $+/-0.2$
\end{tabular}

\section{Expected Casualty Calculation}

The risk due to a sUAS crash on the general population was quantified by using the expected number of casualties, as discussed in Section II.C. In order to do so, the $E_{c}$ components, population density, $\rho_{\text {pop }}$, and Casualty Area, $A_{c}$, need to be modeled.

\section{LaRC Population Density}

Unlike traditional manned aircraft, probability of injury or fatality due to sUAS crash is highly dependent on the operating location of the aircraft. ${ }^{5}$ Within the context of the case study, the population density of NASA LaRC within the vicinity of the planned trajectory was modeled to quantify the expected number of casualties in case of a crash. In order to do so, an estimated number of occupants for each building was documented. Next, the latitude and longitude coordinates were obtained from the NASA LaRC Geographic Information System (GIS) database. The information was then combined to estimate population density in and around each building, in parking lots, and on walkways since the model allows assigning a percentage of population to be out in the open. Figure 3 shows the population density model of the buildings around the planned aircraft trajectory.
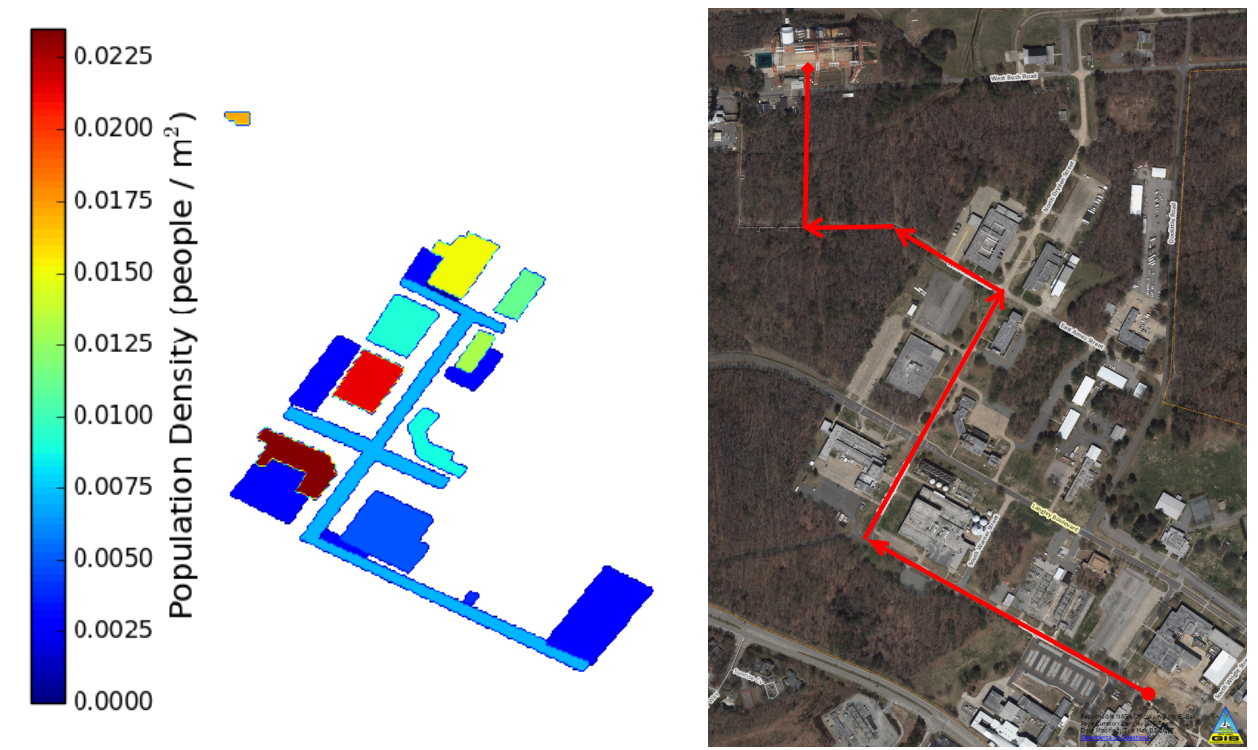

(a) Modeled Population Density Around the Trajec- (b) Aircraft Trajectory and Satellite View tory. of the Area. (Source: NASA GIS Team)

Figure 3. NASA LaRC Population Density Model and Planned Trajectory.

\section{Potential Impact and Casualty Areas}

As there are no passengers to consider, the determination of the potential impact and the casualty areas associated with a failed UAS play a crucial role in determining the expected number of casualties. Figure 4 
illustrates the concept of the casualty and potential impact areas. The potential impact area is defined by any location on the ground where the aircraft could crash. In this case study, the IPP module was employed to determine the size and location of the potential impact area considering trajectory and presence of wind. Additionally, for simplicity, the potential impact area was assumed to have a uniform distribution (i.e., the vehicle will impact any location inside the circle with the same probability). The casualty area is depicted with a red rectangle within the potential impact area and it is assumed that any person inside is considered a casualty given that the vehicle has enough kinetic energy on impact. The casualty area considers the aircraft wingspan, glide/impact angle, and representative characteristics of a person, as discussed in the next sections.

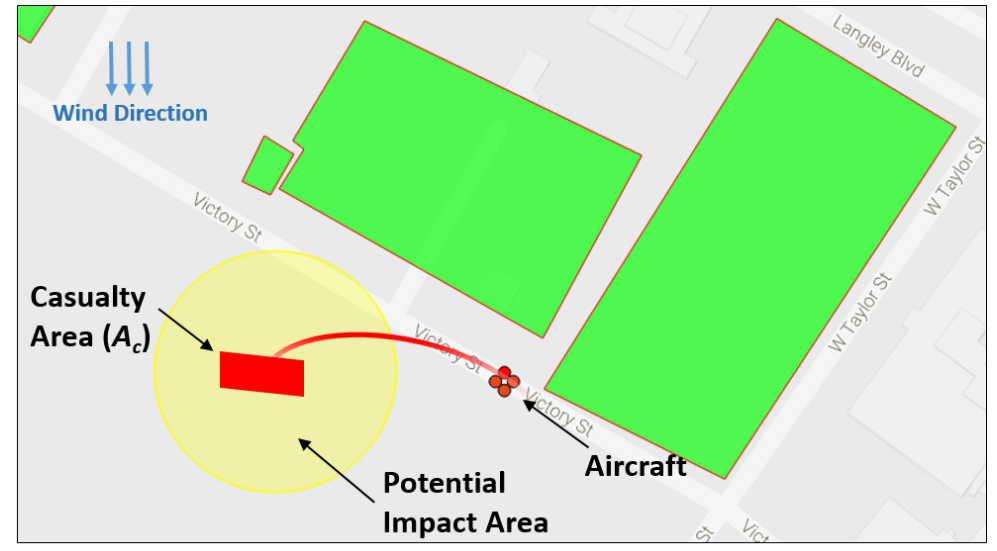

Figure 4. Representative Potential Impact and Casualty Areas. (Source: NASA GIS Team)

\section{3. $\quad$ Sheltering Effects}

The expected casualty calculation takes into consideration people in different sheltering categories. Previous work done in the range safety analysis community was leveraged to provide vulnerability models to estimate risks to people inside buildings. The Range Safety Group ${ }^{28}$ proposed employing the building classes given in Table 6 for their vulnerability models. The vulnerability models define the relationship between object (e.g., UAS) mass, ballistic coefficient, and the effective casualty area. Figure 5 provides the model used for a class B roof. A more detailed discussion regarding the development and use of the vulnerability models can be found in the FAA Flight Safety Analysis Handbook ${ }^{34}$ and in Safety Design for Operations. ${ }^{35}$

Table 6. Building Classes.

\begin{tabular}{|c|c|}
\hline Roof Class & Description \\
\hline $\mathbf{A}$ & $\begin{array}{l}\text {-Mobile homes and trailers } \\
\text {-Temporary office trailers }\end{array}$ \\
\hline B & $\begin{array}{l}\text {-Single family dwellings } \\
\text {-Duplex and fourplex residential dwellings } \\
\text {-Small condominiums and townhouses } \\
\text {-Small apartment buildings }\end{array}$ \\
\hline $\mathrm{C}$ & $\begin{array}{l}\text {-Small retail commercial buildings (gas } \\
\text { stations, stores, restaurants, strip malls) } \\
\text {-Small office and medical office buildings }\end{array}$ \\
\hline D & $\begin{array}{l}\text {-Public buildings (large office buildings, } \\
\text { shopping malls, and apartment complexes, hotels, etc). } \\
\text {-Warehouses } \\
\text {-Manufacturing plants }\end{array}$ \\
\hline
\end{tabular}




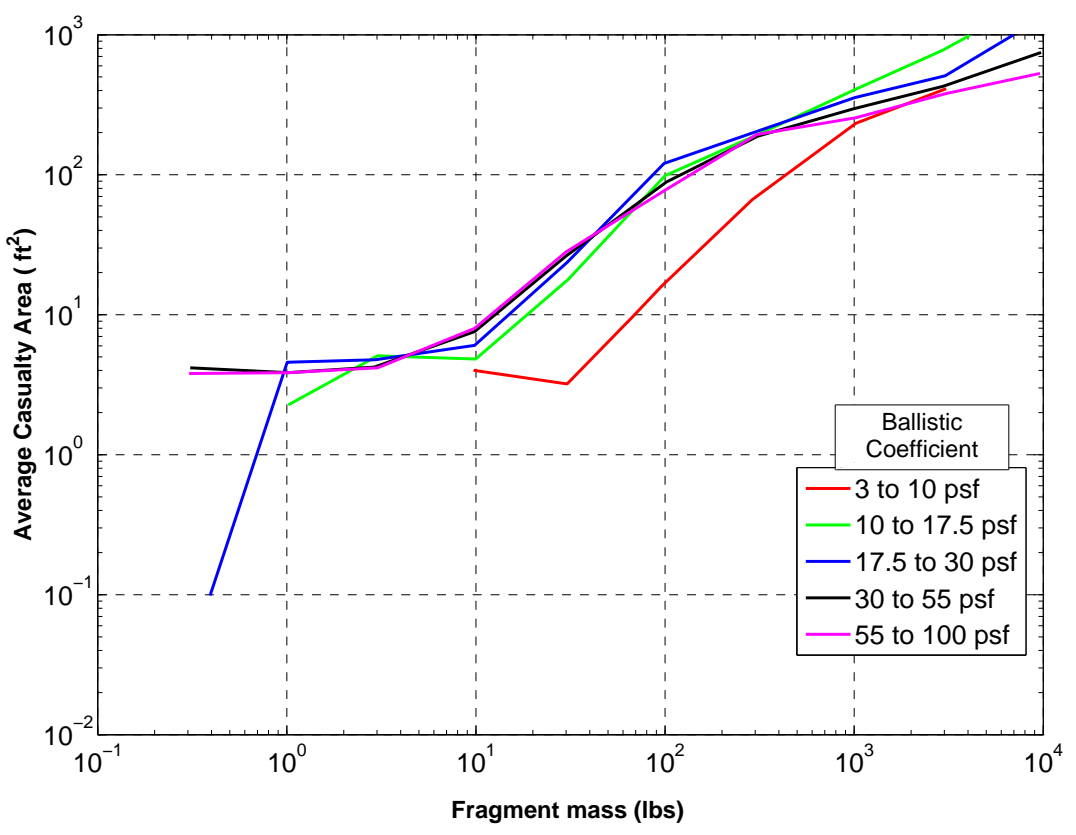

Figure 5. Representative Vulnerability Model for Class B Roof Type.

\section{Casualty Estimation}

Equation (1) needs to be expanded to take into consideration the probability of impact and different sheltering categories. Given the population density map for NASA LaRC, we discretized the potential impact area into $\mathrm{N}$ grid cells. The expected number of casualties for the $j$ th sheltering category is given by:

$$
E_{c_{j}}=\sum_{k=1}^{N} P_{I_{k}} A_{c_{j}} \rho_{\text {pop }_{j}}
$$

where $P_{I_{k}}$ is the probability of impacting the $k^{t h}$ grid cell. $A_{c_{j}}$ and $\rho_{\mathrm{pop}_{j}}$ are the casualty area and population density for the $j^{\text {th }}$ sheltering category. Finally, the expected number of casualties given that there is a failure resulting in a crash is given by:

$$
E_{c_{T}}=\sum_{j=1}^{J} E_{c_{j}}
$$

The vulnerability models discussed in the previous section provide the casualty area given the mass of the vehicle and ballistic coefficient. The expression used to compute the casualty area for people in the open is given by:

$$
A_{c}=\left(w_{\text {span }}+2 R_{p}\right)\left(L_{\text {uav }}+\frac{H_{p}}{\tan \gamma}+2 R_{p}\right)
$$

where $w_{\text {span }}$ represents the wing span, $R_{p}$ is the radius of a person, $L_{\text {uav }}$ is the length of the $\mathrm{UAV}, H_{p}$ is the height of a person, and $\gamma$ represents the glide angle. This expression has been previously introduced by Clothier et al. ${ }^{7}$ and Lum et al. ${ }^{5}$ Employing the methods above, the flight mission described in III.A was simulated. The results for this simulated mission are discussed in the next section.

\section{E. Case Study Details and Results}

The Mission Planner log file for the aforementioned flight mission was employed to simulate a predetermined list of scenarios in order to showcase the risk assessment software capabilities. The simulated scenario variables included power system glitches, presence of high winds, and a fire drill that caused the occupants to evacuate a building on the vehicle's flight path. Figure 6 depicts the ground control station computer 
representation of the software, indicating the UAS location, potential impact area, and assessed risk of unpowered descent considering the population density on the ground.

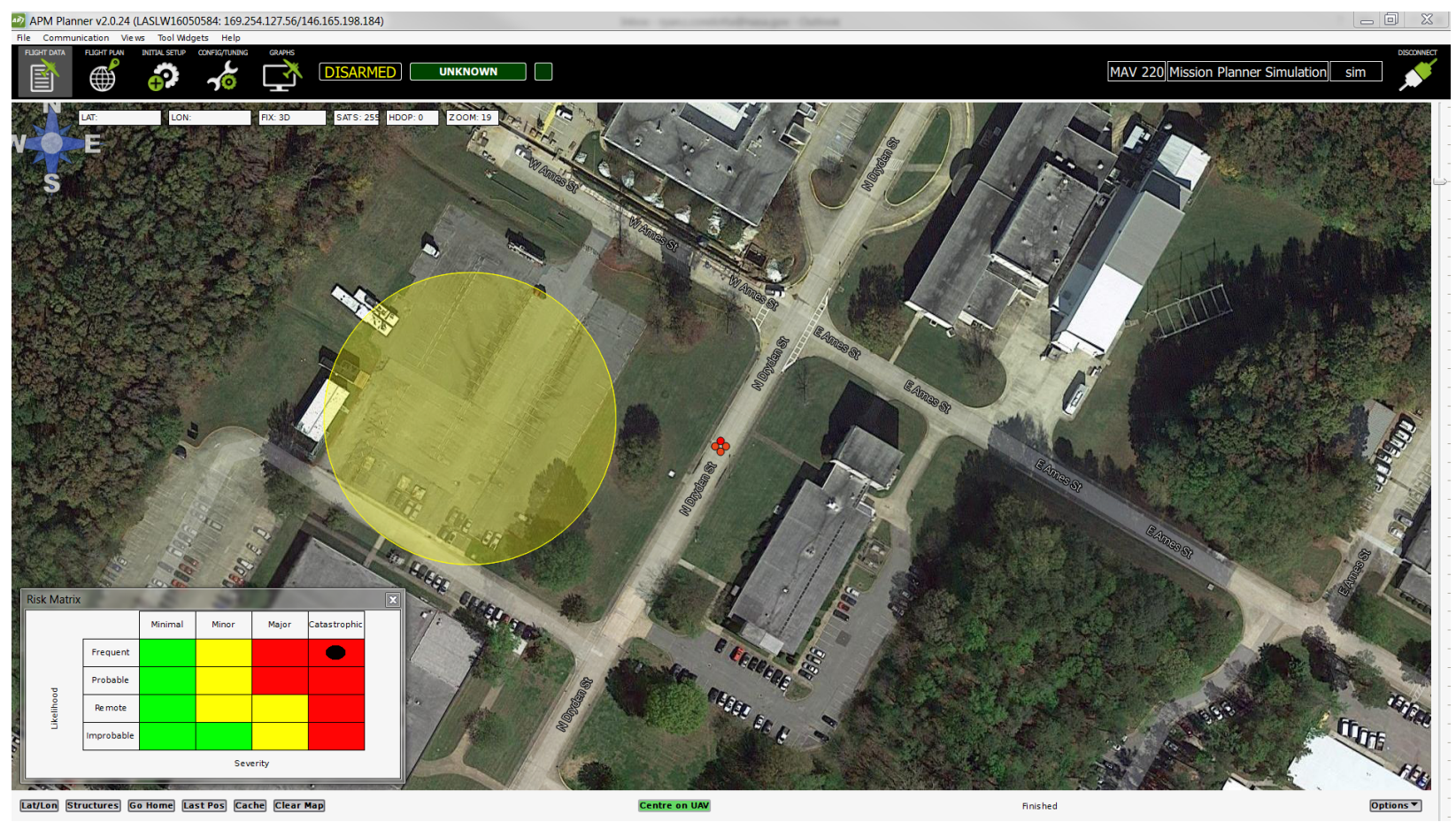

Figure 6. Snapshot of the Ground Control Station Output.

Figure 7 outlines the mishap probability, $E_{c}$ value, and resultant risk level for the duration of the simulated flight. The first failure (depleting battery capacity and lower battery voltage) was introduced around $t=200$ seconds, which caused the unpowered descent probability to increase to $80 \%$ for around one minute. From $t=260$ seconds to $t=580$ seconds, the battery voltage and remaining battery leveled off at an acceptable level. Meanwhile, the $E_{c}$ value stayed below 0.1 (severity index of [0]), which indicated that in the event of a crash, the consequences would be minimal irrespective of KE or mishap probability. For that reason, during the first simulated failure, the flight did not pose any risk to humans on the ground.

The next simulated failure (at $t=581 \mathrm{~s}$ ) brought the remaining battery and battery voltage values to the limit of acceptable thresholds, causing the mishap likelihood to rise to 0.85. Additionally, the winds were substantially increased $(25 \mathrm{~m} / \mathrm{s}$ at 90 degrees), drifting the potential impact area towards a simulated outdoor activity (e.g, fire drill, outdoor gathering, etc.). This caused the $E_{c}$ to rise gradually to over 0.8 (severity index of [4]). The snapshot given in Fig. 6 represents the simulated failure, increased wind presence, and wind impact on aircraft impact point drift while the aircraft is flying along the trajectory at $t=650$. The combined effects of high probability mishap (unpowered descent due to power system failure), higher $E_{c}$ (increased population density without protection of sheltering), and the presence of winds (affecting the flight controllability, increasing the $\mathrm{KE}$ on impact, and causing the aircraft to drift towards populated area) quickly escalated the flight risk from low to medium to high as shown in Fig. 6. 


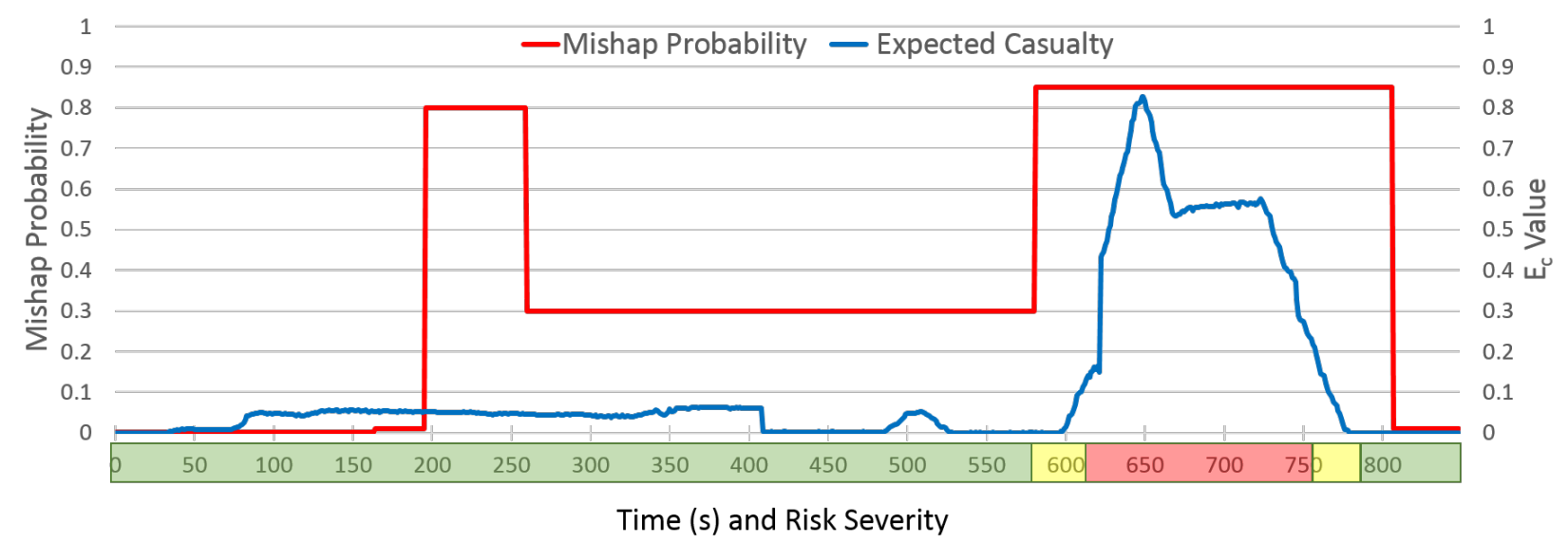

Figure 7. Simulated Model Output, Expected Casualty/Mishap Probability/Risk levels vs. Time

\section{Future Work}

\section{A. Planned Improvements}

The primary focus of the case study was to assess the feasibility of the URAF methodology. The future work involves the implementation of planned improvements previously given Table 4 . The authors believe that the most valuable improvement to the current software would be the inclusion of dynamic population distributions. Future research will explore opportunities associated with fusing the census demographic information with data provided by cellular network towers to obtain a high-resolution augmented population density and distribution capability. Also, by extracting additional aircraft health parameters, not only the dynamic mishap probability estimation can be drastically enhanced but also other failure modes and mishaps can be modeled. Similarly, the prediction of impact point accuracy can be improved by higher fidelity models like ballistics methods and better estimation of aerodynamic parameters via wind tunnel testing.

\section{B. Validation Efforts}

It is crucial to determine the accuracy of predictive models. However, validating the casualties associated with UAS crashes is impractical due to a lack of data on historical accidents. Although Belcastro et al. ${ }^{3}$ reviewed a sizable database concerned with sUAS mishaps, any meaningful statistical inference is unlikely due to the vast variety of aircraft and operational contexts. The literature review indicated that Melnyk et al. ${ }^{13}$ proposed employing bystander fatality rates for General Aviation (GA) accidents by adjusting the model parameters to reflect a typical GA aircraft. Their analysis suggested that model casualty results were representative of casualty rates obtained by historical data. To this point, the authors have not pursued validation of the URAF methodology given the demonstrated case study was solely used to gauge the feasibility of the approach. However, future versions featuring high-fidelity models will be evaluated against historical data, as demonstrated by Melynk et al.

\section{Conclusions}

As projected demand for unmanned aircraft operations increases, risk identification and assessment will continue to play an important role in developing the procedures and protocols necessary for widespread adoption of these systems. NASA's UTM research initiative aims to enable low-altitude civil UAS operations while ensuring safety of the people in the air and on the ground. The framework presented in this paper provides guidance to develop a series of tools that are capable of providing real-time risk assessment for sUAS operations. Leveraging past third party risk assessment studies, the framework proposes the use of aircraft generated health monitoring data along with augmented population density and other dynamic environmental inputs to evaluate casualty risk and inform the operator of imminent failures. Besides serving 
as a ground operator support role, the proposed real-time risk assessment capability can be embedded into UTM services or be used to evaluate mitigation strategies and manage contingencies. A simplified case study was used to demonstrate the feasibility of the concept. By extending the analysis to include other mishaps while taking advantage of higher-fidelity trajectory and casualty estimation methods, the framework has the potential to assist regulators in crafting requirements and applicable safety standards for large scale sUAS operations.

\section{Acknowledgments}

Authors would like to recognize the support of UTM Safety \& Risk Focus team members as well the contributions of John J. Remmes from Simulation Development \& Analysis Branch at NASA Langley Research Center for providing flight data support.

\section{References}

\footnotetext{
${ }^{1}$ Kopardekar, P., Rios, J., Prevot, T., Johnson, M., Jung, J., and III, J. E. R., "Unmanned Aircraft System Traffic Management (UTM) Concept of Operations," 16th AIAA Aviation Technology, Integration, and Operations (ATIO) Conference, No. AIAA 2016-3292, American Institute of Aeronautics and Astronautics, June 2016.

${ }^{2}$ Federal Aviation Administration, "Summary of Small Unmanned Aircraft Rule (Part 107)," June 2016.

${ }^{3}$ Belcastro, C. M., Newman, R. L., Evans, J. K., Klyde, D. H., Barr, L. C., and Ancel, E., "Hazards Identification and Analysis for Unmanned Aircraft System Operations," Aviation Technology, Integration, and Operations (ATIO) Conferences, American Institute of Aeronautics and Astronautics, June 2017 (submitted for publication).

${ }^{4}$ Lazatin, J., "A Method for Risk Estimation Analysis for Unmanned Aerial System Operation over Populated Areas," 14th AIAA Aviation Technology, Integration, and Operations (ATIO) Conference, No. AIAA 2014-2284, American Institute of Aeronautics and Astronautics, June 2014.

${ }^{5}$ Lum, C. and Waggoner, B., "A Risk Based Paradigm and Model for Unmanned Aerial Systems in the National Airspace," Infotech@Aerospace Conferences, No. AIAA-2011-1424, American Institute of Aeronautics and Astronautics, March 2011.

${ }^{6}$ Aalmoes, R., Cheung, Y. S., Sunil, E., Hoekstra, J. M., and Bussink, F., "A Conceptual Third Party Risk Model for Personal and Unmanned Aerial Vehicles," 2015 International Conference on Unmanned Aircraft Systems (ICUAS), June 2015, pp. 1301-1309.

${ }^{7}$ Clothier, R. A., Palmer, J. L., Walker, R. A., and Fulton, N. L., "Definition of Airworthiness Categories for Civil Unmanned Aircraft Systems (UAS)," 27th International Congress of the Aeronautical Sciences, ICAS, June 2010.

${ }^{8}$ Lum, C., Gauksheim, K., Deseure, C., Vagners, J., and McGeer, T., "Assessing and Estimating Risk of Operating Unmanned Aerial Systems in Populated Areas," 11th Aviation Technology, Integration, and Operations (ATIO) Conference, No. AIAA-2011-6918, American Institute of Aeronautics and Astronautics, Sept. 2011.

${ }^{9}$ Ford, A. and McEntee, K., "Assessment of the Risk to Ground Population Due to an Unmanned Aircraft In-Flight Failure," 10th AIAA Aviation Technology, Integration, and Operations (ATIO) Conference, No. AIAA-2010-9056, American Institute of Aeronautics and Astronautics, Sept. 2010.

${ }^{10}$ Dalamagkidis, K., Valavanis, K. P., and Piegl, L. A., "Evaluating the Risk of Unmanned Aircraft Ground Impacts," 16th Mediterranean Conference on Control and Automation, No. 978-1-4244-2505-1/08, IEEE, pp. 709-716.

${ }^{11}$ Weibel, R. and Hansman, R. J., "Safety Considerations for Operation of Different Classes of UAVs in the NAS," 4th Aviation Technology, Integration and Operations (ATIO) Conference, No. AIAA-2004-6421, American Institute of Aeronautics and Astronautics, Sept. 2004.

${ }^{12}$ Burke, D. A., Hall, C. E., and Cook, S. P., "System-Level Airworthiness Tool," Journal of Aircraft, Vol. 48, No. 3, 2011, pp. $777-785$.

${ }^{13}$ Melnyk, R., Schrage, D., Volovoi, V., and Jimenez, H., "A Third-Party Casualty Risk Model for Unmanned Aircraft System Operations," Reliability Engineering 83 System Safety, Vol. 124, 2014, pp. 105-116.

${ }^{14}$ Ancel, E., Shih, A. T., Jones, S. M., Reveley, M. S., Luxhoj, J. T., and Evans, J. K., "Predictive Safety Analytics: Inferring Aviation Accident Shaping Factors and Causation," Journal of Risk Research, Vol. 18, No. 4, April 2015, pp. $428-451$.

${ }^{15}$ Luxhoj, J. T., "A Conceptual Object-Oriented Bayesian Network (OOBN) for Modeling Aircraft Carrier-Based UAS Safety Risk," Journal of Risk Research, Vol. 18, No. 10, Nov. 2015, pp. 1230-1258.

${ }^{16}$ Fenton, N. and Neil, M., Risk Assessment and Decision Analysis with Bayesian Networks, CRC Press, Boca Raton, FL, 2012.

${ }^{17}$ Ancel, E. and Shih, A., "The Analysis of the Contribution of Human Factors to the In-flight Loss of Control Accidents," Aviation Technology, Integration, and Operations (ATIO) Conference, No. AIAA-2012-5548, American Institute of Aeronautics and Astronautics, Sept. 2012.

${ }^{18}$ Washington, A., Clothier, R. A., and Williams, B. P., "A Bayesian Approach to System Safety Assessment and Compliance Assessment for Unmanned Aircraft Systems," Journal of Air Transport Management, Vol. 62, July 2017, pp. 18-33.

${ }^{19}$ Oztekin, A. and Luxhoj, J. T., "Hazard, Safety Risk and Uncertainty Modeling of the Integration of Unmanned Aircraft Systems into the National Airspace," 26th International Congress of the Aeonautical Sciences, ICAS, June 2008.

${ }^{20}$ Hugin Expert, "Hugin Developer, Version. 8.4," Aalborg, Denmark, 2017.

${ }^{21}$ Ancel, E. and Shih, A. T., "Bayesian Safety Risk Modeling of Human-Flightdeck Automation Interaction," NASA TM2015-218791, Aug. 2015.
} 
${ }^{22}$ Barr, L. C., Newman, R. L., Ancel, E., Evans, J. K., Foster, J. V., and Belcastro, C. M., "Preliminary Risk Assessment for Small Unmanned Aircraft Systems," Aviation Technology, Integration, and Operations (ATIO) Conferences, American Institute of Aeronautics and Astronautics, June 2017 (submitted for publication).

${ }^{23}$ Clothier, R. A. and Walker, R. A., Safety Risk Management of Unmanned Aircraft Systems, Springer Netherlands, Dordrecht, 2015, pp. 2229-2275.

${ }^{24}$ Foster, J. V. and Hartman, D. C., "High-Fidelity Multi-Rotor Unmanned Aircraft System Simulation Development for Trajectory Prediction Under Off-Nominal Flight Dynamics," Aviation Technology, Integration, and Operations (ATIO) Conferences, American Institute of Aeronautics and Astronautics, June 2017 (to be presented).

${ }^{25}$ D'Souza, S. N., "Developing a Generalized Trajectory Modeling Framework for Small UAS Performance in the Presence of Wind," Information Systems - AIAA Infotech @ Aerospace, No. AIAA-2017-0447, American Institute of Aeronautics and Astronautics, Jan. 2017.

${ }^{26}$ Russell, C., Jung, J., Willink, G., and Glasner, B., "Wind Tunnel and Hover Performance Test Results for Multicopter UAS Vehicles," American Helicopter Society (AHS) 72nd Annual Forum, May 2016.

${ }^{27}$ Cunningham, K., Cox, D. E., Foster, J. V., Riddick, S. E., and Laughter, S. A., "AirSTAR Beyond Visual Range System Description and Preliminary Test Results," AIAA SciTech Forum, American Institute of Aeronautics and Astronautics, Jan. 2016.

${ }^{28}$ Range Commanders Council, "Standard 321-07 Common Risk Criteria Standards for National Test Ranges: Supplement," Department of Defense, 2007.

${ }^{29}$ Capristan, F. M. and Alonso, J. J., "Range Safety Assessment Tool (RSAT): An Analysis Environment for Safety Assessment of Launch and Reentry Vehicles," 52nd Aerospace Sciences Meeting, No. AIAA 2014-0304, American Institute of Aeronautics and Astronautics, Jan. 2014.

${ }^{30}$ Haimes, Y. Y., Risk Modeling, Assessment, and Management, Wiley, 4th ed., 2015.

${ }^{31}$ Federal Aviation Administration, "Safety Management System (SMS) Manual Version 4.0," No. FAA-H-8083-2, 2014.

${ }^{32}$ DJI, "S1000 Specifications," http://www.dji.com/spreading-wings-s1000/spec, [cited April 23, 2017].

${ }^{33}$ ArduPilot, "APM Planner v2.0," http://ardupilot.org/planner2, [cited April 2, 2017].

${ }^{34}$ Federal Aviation Administration, "Flight Safety Analysis Handbook Version 1.0," 2011.

${ }^{35}$ Allahdadi, F., Rongier, I., Wilde, P., and Sgobba, T., editors, Safety Design for Space Operations, Elsevier Science, 2013. 